\title{
26 Research Square \\ Directed differentiation of human pluripotent stem cells into intermediate mesoderm
}

Kenji Osafune ( $\square$ osafu@cira.kyoto-u.ac.jp )

Osafune Lab (Kyoto University)

\section{Shin-Ichi Mae}

Osafune Lab (Kyoto University)

\section{Method Article}

Keywords: Human pluripotent stem cells, Intermediate mesoderm, Kidney regeneration

Posted Date: September 18th, 2013

DOI: https://doi.org/10.1038/protex.2013.075

License: (c) (i) This work is licensed under a Creative Commons Attribution 4.0 International License. Read Full License 


\section{Abstract}

The induction of kidney lineage cells from human pluripotent stem cells \(hPSCs) has been previously described in some reports, but there have been no protocols so far for the robust induction of intermediate mesoderm $\backslash(\mathrm{IM})$, an early embryonic germ layer that gives rise to kidneys. In the present study, we have established a robust induction protocol using combinational treatment of growth factors, which produces up to $90 \%$ IM cells expressing the IM-specific marker OSR1. This novel method thus supplies an unprecedented system to elucidate the mechanisms of IM development and potentially provide a cell source for kidney regeneration.

\section{Introduction}

The kidneys have highly differentiated and complicated structures, and play critical roles in many physiological processes. Once damaged, kidneys rarely recover their functions. To overcome this problem, kidney regeneration using embryonic stem cells $\backslash$ (ESCs) 1-3 or induced pluripotent stem cells $\backslash$ (iPSCs)4-6, which have an unlimited self-renewal capability and the potential to differentiate into any cell type in the body, is a valuable alternative. Kidneys are derived from IM, and Odd-skipped related $1 \backslash$ (Osr1) is one of the earliest markers specific for IM, although the expression also extends into the lateral plate mesoderm at early stages in mouse, chick and fish embryos7-9. The differentiation of PSCs into 0SR1expressing IM cells is the first step toward the induction of renal lineage cells 10 . In this protocol, we describe how to efficiently induce OSR1+ IM cells from hPSCs.

\section{Reagents}

$\nabla S N L$ feeder cells $\backslash$ (Available from Dr. Allan Bradley of the Sanger Institute or European Collection of Cell Cultures) $\triangle$ hPSCs $\triangle$ Primate ES medium $\backslash($ ReproCELL, cat. no. RCHEMD001) $\otimes$ DMEM $\backslash$ (Nacalai Tesque, cat. no. 08459-35) $\otimes D M E M / F 12+G l u t a m a x \backslash($ Invitrogen, cat. no. 10565-018) $\otimes$ FBS $\backslash($ HyClone, cat. no. SH30070.03) $\otimes$ Knockout serum replacement $\backslash(K S R$, Invitrogen, cat. no. 10828) $\otimes$ Penicillin/streptomycin $\backslash$ (Invitrogen, cat. no. 15140-122) $₫$ Non-essential amino acids $\backslash($ Invitrogen, cat. no. 11140-050) $₫ 2$ mercaptoethanol \(Invitrogen, cat. no. 21985-023) $\ 0.25 \%$ trypsin $\backslash($ Invitrogen, cat. no. 25200-056) $\otimes 0.1 \%$ collagenase IV \(Invitrogen, cat. no. 17104-019) $\otimes \mathrm{CaCl} 2 \backslash($ Nacalai Tesque, cat. no. 08894-25) $\otimes$ Accutase $\backslash($ Innovative Cell Technologies, Inc., cat. no. AT104) $\otimes$ PBS \(-) \(Nacalai Tesque, cat. no. 14249-95) $\square$ Recombinant human basic fibroblast growth factor $\backslash(\mathrm{bFGF}$, Wako, cat. no. 064-04541) $\otimes Y 27,632 \backslash$ (Wako, cat. no. 253-00513) $\otimes$ Recombinant human/mouse/rat activin A (R\&D Systems, cat. no. 338-AC) Recombinant mouse Wnt3a \(R\&D Systems, cat. no. 1324-WN) $\otimes$ CHIR99021 (Wako, cat. no. 039-20831) $\otimes$ Recombinant human BMP7 \(R\&D Systems, cat. no. 354-BP) $\nabla$ Matrigel $\backslash(B D$, cat. no. 354230) $\nabla$ Human Collagen Type I (BD, cat. no. 354265) $\otimes$ Gelatin, from porcine skin \(SIGMA, cat. no. G1890) All media should be sterilized by $0.22 \mu \mathrm{m}$ filtration. $\triangle \mathrm{hPSC}$ medium for maintenance culture Primate ES medium supplemented with $500 \mathrm{U} / \mathrm{ml}$ penicillin/streptomycin and $4 \mathrm{ng} / \mathrm{ml}$ recombinant human bFGF $\otimes$ Stage 1 medium DMEM/F12+Glutamax supplemented with $500 \mathrm{U} / \mathrm{ml}$ penicillin/streptomycin and 2\% FBS $\otimes$ Stage 2 medium DMEM/F12+Glutamax supplemented with $0.1 \mathrm{mM}$ non-essential amino acids, $500 \mathrm{U} / \mathrm{ml}$ 
penicillin/streptomycin, $0.55 \mathrm{mM}$ 2-mercaptoethanol and $10 \% \mathrm{KSR} \otimes \mathrm{CTK}$ dissociation solution PBS $\backslash(-)$ supplemented with $0.25 \%$ trypsin, $0.1 \%$ collagenase IV, $20 \% \mathrm{KSR}$ and $1 \mathrm{mM} \mathrm{CaCl} 2$

\section{Equipment}

$\triangle \mathrm{CO} 2$ incubator with controlling and monitoring system for $\mathrm{CO} 2$, humidity and temperature $\otimes$ Biosafety cabinet for cell culture $₫$ Cell culture centrifuge $\otimes$ Cell culture disposables: Petri dishes, multi-well plates, centrifuge tubes, pipettes, pipette tips, and cell scrapers

\section{Procedure}

Overview Steps 1 - 9: Maintenance of hPSCs Steps 10 - 22: Induction of IM cells by embryoid body $\backslash$ (EB) formation \(EB method) Steps 23 - 29: Induction of IM cells by colony formation \(Colony method) Steps 30 - 40: Induction of IM cells with single cell dissociation \(Single cell method) Maintenance of hPSCs Preparation of SNL feeder cells 1 . Partially thaw the frozen vial of mitotically inactivated SNL feeder cells in $37^{\circ} \mathrm{C}$ water bath, resuspend the $1 \mathrm{ml}$ cell solution in $10 \mathrm{ml} \mathrm{DMEM} / 10 \%$ FBS medium, and spin down at approximately $160 \mathrm{~g}$ for $5 \mathrm{~min}$. 2. Remove the supernatant and resuspend the pellet in $10 \mathrm{ml}$ DMEM/10 \% FBS medium. 3. Plate the cell suspension onto gelatin-coated plates at a density of approximately 50,000 cells/cm2 and incubate overnight. Passage of hPSCs 4 . Remove differentiated hPSCs using aseptic technique. 5. Aspirate old hPSC medium from plate, wash with $5 \mathrm{ml}$ PBS $\backslash(-)$, and add warm CTK dissociation solution $\backslash(1 \mathrm{ml} / 10 \mathrm{~cm}$ plate $)$. 6 . Incubate at $37^{\circ} \mathrm{C}$ for $3 \mathrm{~min}$. 7 . Wash out SNLs twice with $5 \mathrm{ml}$ PBS $\backslash(-)$. 8. Harvest the hPSC colonies in $5 \mathrm{ml}$ fresh hPSC medium by using a cell scraper. 9. Pipette and triturate the hPSC colonies in hPSC medium using P1000 pipetman and split onto new feeder cells at a ratio between 1:3 and 1:6. Induction of IM cells by embryoid body $\backslash(\mathrm{EB})$ formation $\backslash$ (EB method) 10. Repeat steps $4-7.11$. Add $5 \mathrm{ml}$ hPSC medium and scrape hPSCs off the surface with a cell scraper, then pipet the cells up and down 5-10 times using P1000 pipetman. 12. Plate the suspension onto gelatin-coated plate and incubate at $37^{\circ} \mathrm{C}$ for $30 \mathrm{~min}$ to remove SNLs. 13. Transfer the hPSC cell solution to $15 \mathrm{ml}$ tube and allow cells to settle via gravity. 14 . Remove the supernatant and resuspend with $10 \mathrm{ml}$ Stage 1 medium, then allow cells to settle via gravity. 15. Remove the supernatant and resuspend with $8 \mathrm{ml}$ Stage 1 medium containing $100 \mathrm{ng} / \mathrm{ml}$ recombinant human/mouse/rat activin A and $100 \mathrm{ng} / \mathrm{ml}$ recombinant mouse Wnt3a or $3 \mu \mathrm{M}$ CHIR99021. 16. Distribute the cell suspension into a low attachment 6-well plate $\backslash(2 \mathrm{ml} /$ well $)$ to form EB. Culture at $37^{\circ} \mathrm{C}$ for 2 days. 17 . Transfer the EB solution to a $15 \mathrm{ml}$ tube and allow EBs to settle via gravity. 18. Remove the supernatant and resuspend with $10 \mathrm{ml}$ Stage 2 medium, then allow cells to settle via gravity. 19. Remove the supernatant and resuspend with $8 \mathrm{ml}$ Stage 2 medium containing $100 \mathrm{ng} / \mathrm{ml}$ recombinant human BMP7 and $100 \mathrm{ng} / \mathrm{ml}$ recombinant mouse Wnt3a or $3 \mu \mathrm{M}$ CHIR99021. 20. Transfer the cell solution onto a gelatin-coated 6-well plate $\backslash(2 \mathrm{ml} /$ well). 21. Change medium with BMP7 and Wnt3a or CHIR99021 every 2 - 3 days and culture at $37^{\circ} \mathrm{C}$ for 4 - 20 days. Induction of IM cells by colony formation \(Colony method) 22. Repeat steps 10 - 14. 23. Remove the supernatant, resuspend with $12 \mathrm{ml}$ SNL-conditioned hPSC medium containing 10 $\mathrm{ng} / \mathrm{ml}$ recombinant human bFGF and seed on Matrigel-coated 24-well plate $\backslash(500 \mu \mathrm{l} /$ well). 24. Grow the 
cells until hPSC colonies reach approximately $70 \%$ confluency. 25 . Aspirate the medium and wash twice with PBS $\backslash(-)$. 26. Add Stage 1 medium containing $100 \mathrm{ng} / \mathrm{ml}$ recombinant human/mouse/rat activin A and $100 \mathrm{ng} / \mathrm{ml}$ recombinant mouse Wnt3a or $3 \mu \mathrm{M}$ CHIR99021 $\backslash(500 \mu \mathrm{l} /$ well $)$ and culture at $37^{\circ} \mathrm{C}$ for 2 days. 27. Aspirate the medium and wash with Stage 2 medium. 28. Add Stage 2 medium containing 100 $\mathrm{ng} / \mathrm{ml}$ recombinant human BMP7 and $100 \mathrm{ng} / \mathrm{ml}$ recombinant mouse Wnt3a or $3 \mu \mathrm{M}$ CHIR99021 \(500 $\mu \mathrm{l} /$ well). 29. Change medium with BMP7 and Wnt3a or CHIR99021 every 2 - 3 days and culture for 8 days. Induction of IM cells with single cell dissociation \(Single cell method) 30. Repeat steps 4-7. 31 . Add $2 \mathrm{ml}$ warm Accutase. 32. Incubate at $37^{\circ} \mathrm{C}$ for $6 \mathrm{~min}$. 33. Pipet and dissociate the hPSC colonies into single cells using P1000 pipetman. 34 . Add $8 \mathrm{ml}$ Stage 1 medium and pipet the cells up and down using P1000 pipetman. 35. Count the cell number. 36 . Transfer the hPSC cell solution to $15 \mathrm{ml}$ tube and spin down at $160 \mathrm{~g}$ for $5 \mathrm{~min}$. 37. Remove the supernatant and resuspend with Stage 1 medium containing $100 \mathrm{ng} / \mathrm{ml}$ recombinant human/mouse/rat activin A, $100 \mathrm{ng} / \mathrm{ml}$ recombinant mouse Wnt3a or $3 \mu \mathrm{M}$ CHIR99021, and $10 \mu \mathrm{M}$ Y27,632 at a density of $6.0 \times 105 \mathrm{cells} / \mathrm{ml}$. 38. Distribute into Human Collagen Type I-coated 24-well plates at a density of $1.5 \times 105 \mathrm{cells} / \mathrm{cm} 2 \backslash(500 \mu \mathrm{l} /$ well $)$ and culture at $37^{\circ} \mathrm{C}$ for 2 days. 39. Aspirate the medium and wash with warm Stage 2 medium. 40. Add Stage 2 medium containing $100 \mathrm{ng} / \mathrm{ml}$ recombinant human BMP7 and $100 \mathrm{ng} / \mathrm{ml}$ recombinant mouse Wnt3a or $3 \mu \mathrm{M}$ CHIR99021 \(500 $\mu \mathrm{l} /$ well). 41. Change medium with BMP7 and Wnt3a or CHIR99021 every $2-3$ days and culture at $37^{\circ} \mathrm{C}$ for 8 days.

\section{Troubleshooting}

$\backslash$ (a) No formation of EBs - Check the size and the density of hPSC colonies - Do not form smaller EBs Check the concentration of activin A and Wnt3a or CHIR99021 - Check for contamination of Mycoplasma species in hPSCs or SNLs - Change the batch of hPSCs or SNLs $\backslash(b)$ Low attachment of EBs - Check the gelatin-coated plate - Matrigel-coated plate is better for attachment - Check the concentration of BMP7 and Wnt3a or CHIR99021 \(c) Low survival rate of the cells in Colony method - Check the size and the density of hPSC colonies - Check the concentration of activin A, BMP7 and Wnt3a or CHIR99021 - Check for contamination of Mycoplasma species in hPSCs or SNLs - Change the batch of hPSCs or SNLs $\backslash(\mathrm{d})$ Low survival rate of the differentiated cells in Single cell method - Check the plating density of hPSCs - Do not incubate in Accutase for an excessive amount of time during the single cell dissociation step - Check the activity of Y27,632 - Check the concentration of activin A, BMP7 and Wnt3a or CHIR99021 - Check for contamination of Mycoplasma species in hPSCs or SNLs - Change the batch of hPSCs or SNLs

\section{Anticipated Results}

The temporal expression pattern of OSR1 will show a gradual increase, with a peak at culture day 19 with the EB method and at day 11 with the Single cell method $\backslash$ (Figure 1a, b). The Single cell method produces up to $90 \%$ OSR $1+$ cells $\backslash$ (Figure 1c). These human intermediate mesoderm $\backslash(\mathrm{IM})$ cells can differentiate into multiple cell types constituing IM-derived organs, such as kidney, adrenal cortex and gonad, in vitro $\backslash$ (Figure 2). 


\section{References}

1. Evans, M.J. \& Kaufman, M.H. Establishment in culture of pluripotential cells from mouse embryos. Nature 292, 154-6 \(1981). 2. Martin, G.R. Isolation of a pluripotent cell line from early mouse embryos cultured in medium conditioned by teratocarcinoma stem cells. Proc Natl Acad Sci U S A 78, 7634-8 \ (1981). 3. Thomson, J.A. et al. Embryonic stem cell lines derived from human blastocysts. Science 282, 1145-7 \(1998). 4. Takahashi, K. et al. Induction of pluripotent stem cells from adult human fibroblasts by defined factors. Cell 131, 861-72 \(2007). 5. Takahashi, K. \& Yamanaka, S. Induction of pluripotent stem cells from mouse embryonic and adult fibroblast cultures by defined factors. Cell 126, 663-76 \(2006). 6. $\mathrm{Yu}, \mathrm{J}$. et al. Induced pluripotent stem cell lines derived from human somatic cells. Science 318, 1917-20 I (2007). 7. Tena, J.J. et al. Odd-skipped genes encode repressors that control kidney development. Dev Biol 301, 518-31 \(2007). 8. James, R.G., Kamei, C.N., Wang, Q., Jiang, R. \& Schultheiss, T.M. Odd-skipped related 1 is required for development of the metanephric kidney and regulates formation and differentiation of kidney precursor cells. Development 133, 2995-3004 \(2006). 9. Mugford, J.W., Sipila, P., McMahon, J.A. \& McMahon, A.P. Osr1 expression demarcates a multi-potent population of intermediate mesoderm that undergoes progressive restriction to an Osr1-dependent nephron progenitor compartment within the mammalian kidney. Dev Biol 324, 88-98 \(2008). 10. Mae, S. et al. Combination of small molecules enhances differentiation of mouse embryonic stem cells into intermediate mesoderm through BMP7-positive cells. Biochem Biophys Res Commun 393, 877-82 \(2010).

\section{Acknowledgements}

We thank Drs. T. Toyoda, T. Araoka, T. Toyohara and T. Inoue for helpful discussion. This study was supported in part by research grants from the Leading Project of MEXT, the Uehara Memorial Foundation and the Takeda Science Foundation, by the Japan Society for the Promotion of Science \(JSPS) through its "Funding Program for World-Leading Innovative R\&D on Science and Technology \(FIRST Program)" and Grant-in-Aid for Young Scientists \(B) to K.O., and by Japan Science and Technology Agency $\backslash($ JST) through PRESTO and JST Yamanaka iPS Cell Special Project. S.M. was supported by a fellowship from JSPS.

\section{Figures}



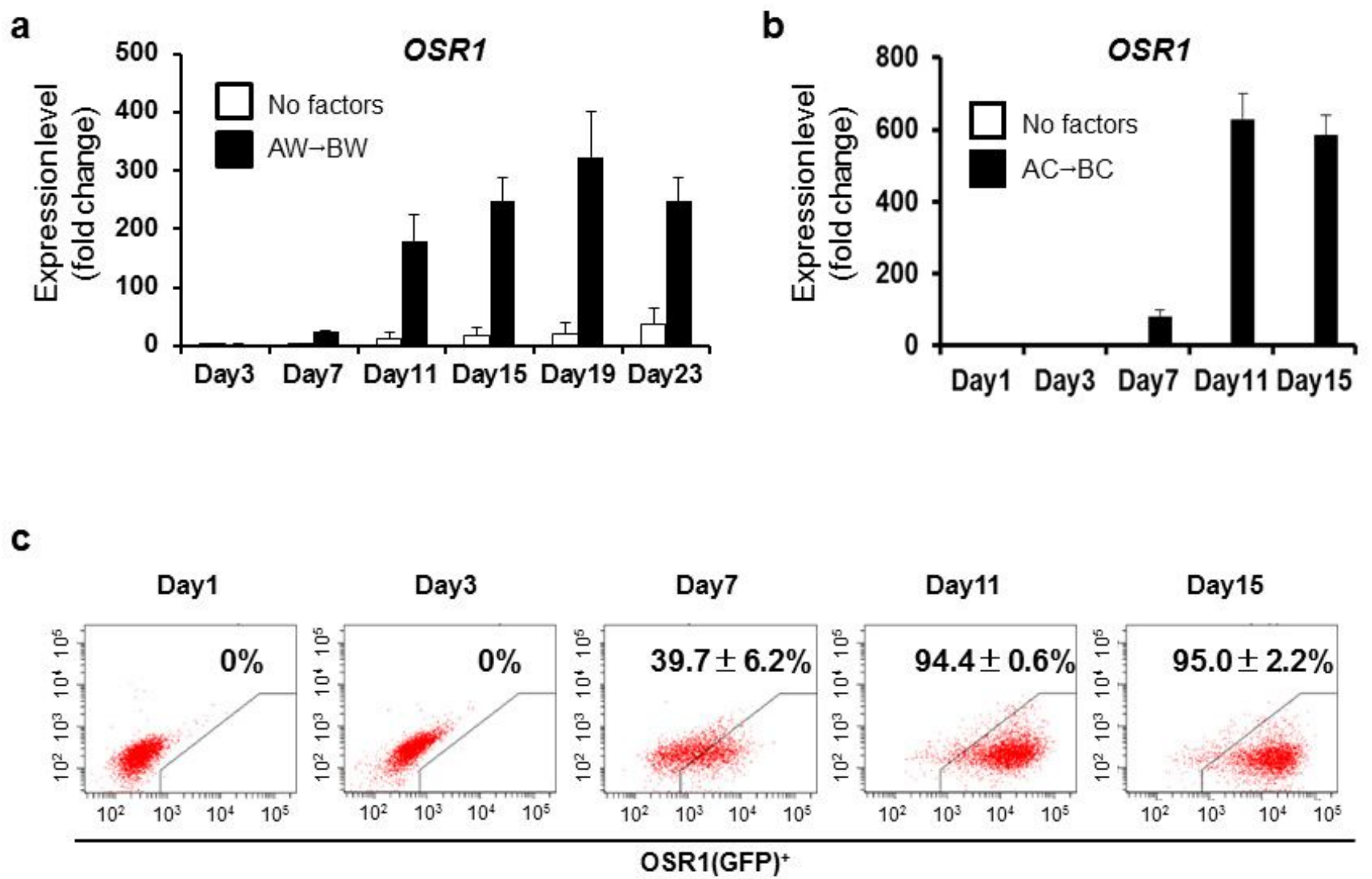

Figure 1

Differentiation of hiPSCs into the intermediate mesoderm (IM). $(a, b)$ The time course analysis of OSR1 expression in the differentiation culture with EB method (a) and Single cell method (b). Each value was normalized to the samples on day 1 before treatments. Note that the OSR 1 expression of samples on culture day 3 in a and on culture days 1 and 3 and without factors in $b$ is so low that the histogram bars are at the baseline. White bars: no factors; black bars $(A W \rightarrow B W)$ : Stage $1,100 \mathrm{ng} / \mathrm{ml}$ activin $A+100$ $\mathrm{ng} / \mathrm{ml}$ Wnt3a, Stage 2, $100 \mathrm{ng} / \mathrm{ml}$ BMP7 $+100 \mathrm{ng} / \mathrm{ml}$ Wnt3a. (AC $\rightarrow B C$ ): Stage 1, $100 \mathrm{ng} / \mathrm{ml}$ activin A +3 $\mu \mathrm{M}$ CHIR99021, Stage 2, $100 \mathrm{ng} / \mathrm{ml} \mathrm{BMP7}+3 \mu \mathrm{M}$ CHIR99021. (c) The temporal differentiation pattern of OSR $1+$ cells using the Single cell method. The data from three independent experiments are presented as the means $\pm S D(n=3)$ in a-c. 
a

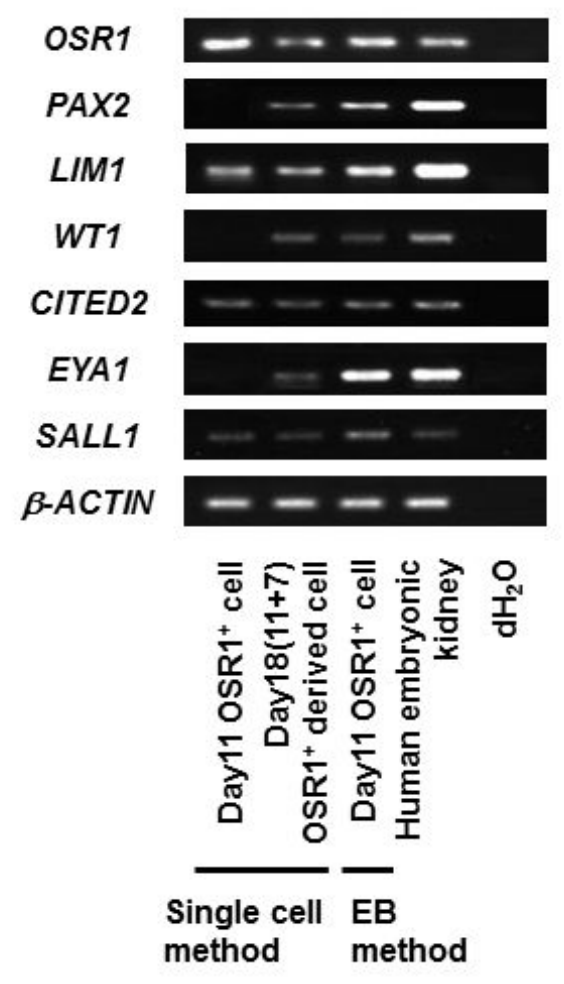

b

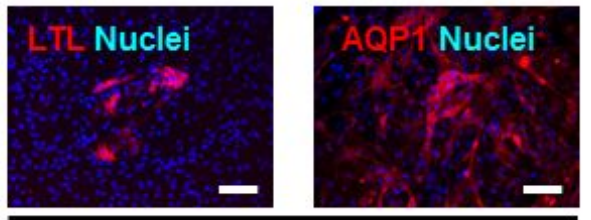

Proximal tubule

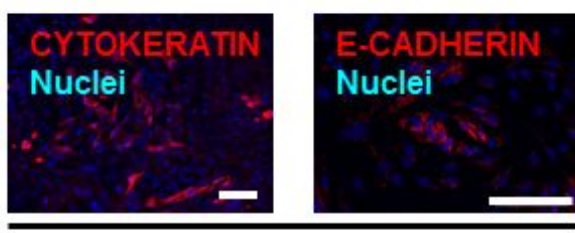

Epithelia

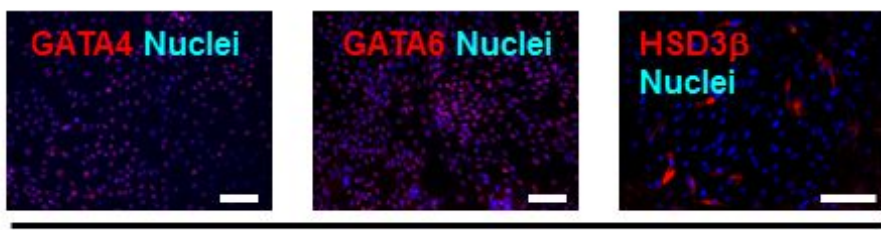

Gonad or Adrenal cortex

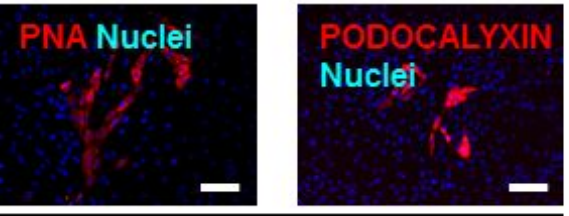

Glomerulus (Podocyte)

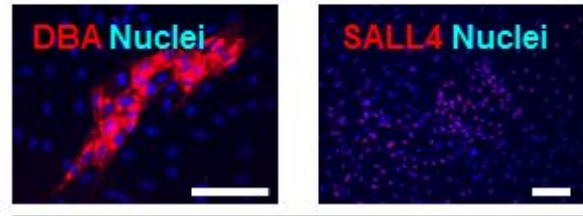

Nephric duct and Ureteric bud

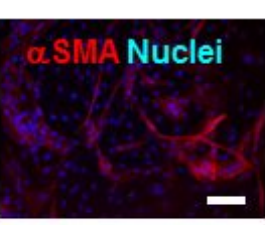

Smooth muscle

\section{Figure 2}

In vitro differentiation of OSR1+ cells into IM derivatives. (a) RT-PCR analyses of marker gene expression for IM or kidney lineage in isolated OSR1+ cells on culture day 11 using two induction protocols (EB and Single cell methods), and the differentiated cells on day 18 (after an additional 7-day culture of the day 11 isolated OSR1 + cells generated with the single cell method). (b) The differentiated cells on day 18 were stained with antibodies or lectins against markers for IM derivatives: Lotus Tetragonobulus lectin (LTL) and Aquaporin (AQP1) for proximal renal tubule, Peanut agglutinin (PNA) and PODOCALYXIN for glomerular podocytes, CYTOKERATIN and E-CADHERIN for renal epithelia, Dolichos biflorus agglutinin (DBA) and SALL4 for nephric duct and ureteric bud, GATA4, GATA6 and HSD3 $\beta$ for the gonads or adrenal cortex, and aSMA for smooth muscle. Scale bars, $100 \mu \mathrm{m}$. 